\title{
APÉRY-LIKE NUMBERS AND FAMILIES OF NEWFORMS WITH COMPLEX MULTIPLICATION
}

\author{
ALEXIS GOMEZ, DERMOT MCCARTHY, DYLAN YOUNG
}

\begin{abstract}
Using Hecke characters, we construct two infinite families of newforms with complex multiplication, one by $\mathbb{Q}(\sqrt{-3})$ and the other by $\mathbb{Q}(\sqrt{-2})$. The values of the $p$-th Fourier coefficients of all the forms in each family can be described by a single formula, which we provide explicitly. This allows us to establish a formula relating the $p$-th Fourier coefficients of forms of different weights, within each family. We then prove congruence relations between the $p$-th Fourier coefficients of these newforms at all odd weights and values coming from two of Zagier's sporadic Apéry-like sequences.
\end{abstract}

\section{Introduction and Statement of Results}

In 1978, Apéry [4] provided quite an unexpected proof of the irrationality of $\zeta(3)$. His methods also yielded another proof of the irrationality of $\zeta(2)$. The sequences

$$
A(n)=\sum_{k=0}^{n}\left(\begin{array}{l}
n \\
k
\end{array}\right)^{2}\left(\begin{array}{c}
n+k \\
k
\end{array}\right), \quad B(n)=\sum_{k=0}^{n}\left(\begin{array}{l}
n \\
k
\end{array}\right)^{2}\left(\begin{array}{c}
n+k \\
k
\end{array}\right)^{2}
$$

arose in those proofs and are now commonly referred to as the Apéry numbers. Since then, there has been substantial interest in both the intrinsic arithmetic properties of the Apéry numbers and their relationship to modular forms. For example, consider the unique newform in $S_{3}\left(\Gamma_{0}(16),\left(\frac{-4}{.}\right)\right)$, which has complex multiplication $(\mathrm{CM})$ by $\mathbb{Q}(\sqrt{-1})$,

$$
h(z)=q \prod_{n \geq 1}\left(1-q^{4 n}\right)^{6}=: \sum_{n=1}^{\infty} \alpha(n) q^{n},
$$

where $q:=e^{2 \pi i z}$ and $z \in \mathbb{H}$. Ahlgren [1] proved that, for all primes $p \geq 5$,

$$
A\left(\frac{p-1}{2}\right) \equiv \alpha(p) \quad\left(\bmod p^{2}\right)
$$

thus confirming a conjecture in [18]. A similar result applies to the $B(n)$ sequence and a newform in $S_{4}\left(\Gamma_{0}(8)\right)$ [2].

Inspired by Apéry, Beukers [5] gave another proof of the irrationality of $\zeta(2)$ and $\zeta(3)$ by considering certain families of integrals which satisfy $\mathbb{Q}$-linear relations with the respective zeta values. The Apéry numbers appear as coefficients in these relations. More recently, Brown [7] introduced cellular integrals which can be expressed as $\mathbb{Q}$-linear combinations of multiple zeta values, and include Beukers integrals as a special cases. In [13, the authors, of whom the second author of this paper is one, examine sequences arising from the coefficients in these linear combinations. They show that all powers of the Apéry numbers

2010 Mathematics Subject Classification. Primary: 11F11, 11B83; Secondary: 11A07. 
are among these sequences and that they too satisfy congruence relations with Fourier coefficients of modular forms which have complex multiplication by $\mathbb{Q}(\sqrt{-1})$, similar to (1.2). To do this, they construct, using Hecke characters, an infinite family of modular forms, which have complex multiplication by $\mathbb{Q}(\sqrt{-1})$, and whose $p$-th Fourier coefficients can be described by a single formula. Specifically, for every positive integer $k \geq 2$, there exists a weight $k \mathrm{CM}$ newform $h_{k}(z)=: \sum_{n=1}^{\infty} \alpha_{k}(n) q^{n} \in S_{k}\left(\Gamma_{0}\left(N_{k}\right), \chi_{k}\right)$ such that

$\alpha_{k}(p)= \begin{cases}(-1)^{\frac{(x+y-1)(k-1)}{2}}\left[(x+i y)^{k-1}+(x-i y)^{k-1}\right], & \text { if } p \equiv 1(\bmod 4), p=x^{2}+y^{2}, x \text { odd, } \\ 0, & \text { if } p \equiv 3(\bmod 4) .\end{cases}$

The level $N_{k}$ and character $\chi_{k}$ are explicitly stated and depend only the congruence class of $k$ modulo 4 . Using this formula for the $p$-th Fourier coefficients, nice congruence relations between Fourier coefficients of forms of different weight can be found. For example,

$$
\alpha_{3}(p)^{s} \equiv \alpha_{t}(p) \quad\left(\bmod p^{2}\right)
$$

where $t=2 s+1$. Then, using (1.2), we get that

$$
A\left(\frac{p-1}{2}\right)^{s} \equiv \alpha_{t}(p) \quad\left(\bmod p^{2}\right)
$$

In this paper, we perform similar exercises with, firstly, a family of modular forms with complex multiplication by $\mathbb{Q}(\sqrt{-3})$ and the sequence

$$
C(n)=\sum_{k=0}^{n}\left(\begin{array}{l}
n \\
k
\end{array}\right)^{2}\left(\begin{array}{c}
2 k \\
k
\end{array}\right)
$$

and then with a family of modular forms with complex multiplication by $\mathbb{Q}(\sqrt{-2})$ and the sequence

$$
D(n)=\sum_{k=0}^{n}\left(\begin{array}{l}
n \\
k
\end{array}\right)^{3}
$$

The sequences $C(n)$ and $D(n)$ are two of the so-called sporadic Apéry-like numbers found by Zagier [20]. We will discuss the Apéry-like numbers and some properties of $C(n)$ and $D(n)$ in Section 2.3 .

The results of this paper are as follows. First, using Hecke characters, we construct an infinite family of newforms which have complex multiplication by $\mathbb{Q}(\sqrt{-3})$ and whose $p$-th Fourier coefficients can be described by a single formula. This is slightly more complicated than the $\mathbb{Q}(\sqrt{-1})$ case, as the ring of integers of $\mathbb{Q}(\sqrt{-3})$ admits half integers and its group of units is larger. Also, we need to consider a larger number of congruence classes for $k$, each of which requires its own construction.

Theorem 1.1. Let $k \geq 2$ be an integer. Then there exists a weight $k$ newform with $C M$ by $\mathbb{Q}(\sqrt{-3})$

$$
f_{k}(z)=: \sum_{n=1}^{\infty} \gamma_{k}(n) q^{n} \in \begin{cases}S_{k}\left(\Gamma_{0}(36)\right), & \text { if } k \equiv 0,2(\bmod 6), \\ S_{k}\left(\Gamma_{0}(3),\left(\frac{-3}{\cdot}\right)\right), & \text { if } k \equiv 1(\bmod 6), \\ S_{k}\left(\Gamma_{0}(12),\left(\frac{-3}{-}\right)\right), & \text { if } k \equiv 3,5(\bmod 6), \\ S_{k}\left(\Gamma_{0}(9)\right), & \text { if } k \equiv 4(\bmod 6) .\end{cases}
$$


Furthermore, for any odd prime $p$,

$$
\gamma_{k}(p)= \begin{cases}(a+b \sqrt{-3})^{k-1}+(a-b \sqrt{-3})^{k-1} & \text { if } p \equiv 1(\bmod 6), \\ (-3)^{\frac{k-1}{2}} & \text { if } p=3 \text { and } k \text { odd } \\ 0 & \text { otherwise, }\end{cases}
$$

where $p=a^{2}+3 b^{2}$ when $p \equiv 1(\bmod 6)$, with $a \equiv 1(\bmod 3)$ and $b>0$ integers. Therefore, for all $n \geq 1, k \geq 2, \gamma_{k}(n)$ is an integer.

We then get some nice relations between the Fourier coefficients of forms of different weights.

Corollary 1.2. Let $k \geq 2$ be an integer and let $f_{k}(z)=: \sum_{n=1}^{\infty} \gamma_{k}(n) q^{n}$ be the weight $k$ $C M$ newform described in Theorem 1.1. Then, for any prime $p>3$ and integer $r \geq 1$,

$\gamma_{k}(p)^{r}=\sum_{t=0}^{\left\lfloor\frac{r-1}{2}\right\rfloor}\left(\begin{array}{l}r \\ t\end{array}\right) p^{t(k-1)} \gamma_{(r-2 t)(k-1)+1}(p)+ \begin{cases}\left(\begin{array}{l}r \\ \frac{r}{2}\end{array}\right) p^{\frac{r}{2}(k-1)}, & \text { if } p \equiv 1(\bmod 6) \text { and } r \text { even, } \\ 0, & \text { otherwise. }\end{cases}$

Also, for $k$ odd,

$$
\gamma_{k}(3)^{r}=\gamma_{r(k-1)+1}(3) .
$$

Corollary 1.3. Let $k \geq 2$ be an integer and let $f_{k}(z)=: \sum_{n=1}^{\infty} \gamma_{k}(n) q^{n}$ be the weight $k$ $C M$ newform described in Theorem 1.1. Then, for any prime $p>3$ and integer $r \geq 1$,

$$
\gamma_{k}(p)^{r} \equiv \gamma_{r(k-1)+1}(p) \quad\left(\bmod p^{k-1}\right) .
$$

Results in [18] provide us with a congruence relation between values of $C(n)$ and the Fourier coefficients of the weight three form, $f_{3}(z)$, from Theorem 1.1. Combining this with Corollary 1.3 we relate values of $C(n)$ to all $f_{k}(z)$ for $k$ odd.

Theorem 1.4. Let $k \geq 2$ be an integer and let $f_{k}(z)=: \sum_{n=1}^{\infty} \gamma_{k}(n) q^{n}$ be the weight $k$ $C M$ newform described in Theorem 1.1. Let $C(n)$ be the sequence defined in (1.5). Then, for any prime $p>3$ and integer $r \geq 1$,

$$
C\left(\frac{p^{r}-1}{2}\right) \equiv \gamma_{2 r+1}(p) \quad(\bmod p) .
$$

We note that the congruence relation in Theorem 1.4 does not hold modulo $p^{2}$.

Next we construct an infinite family of newforms which have complex multiplication by $\mathbb{Q}(\sqrt{-2})$ and establish similar results with respect to the $D(n)$ sequence.

Theorem 1.5. Let $k \geq 3$ be an odd integer. Then there exists a weight $k$ newform with $C M$ by $\mathbb{Q}(\sqrt{-2})$,

$$
g_{k}(z)=: \sum_{n=1}^{\infty} \beta_{k}(n) q^{n} \in S_{k}\left(\Gamma_{0}(8),\left(\frac{-8}{\cdot}\right)\right)
$$

such that for any prime $p$,

$$
\beta_{k}(p)= \begin{cases}(c+d \sqrt{-2})^{k-1}+(c-d \sqrt{-2})^{k-1} & \text { if } p \equiv 1,3(\bmod 8), \\ (-2)^{\frac{k-1}{2}} & \text { if } p=2 \\ 0 & \text { otherwise }\end{cases}
$$


where $p=c^{2}+2 d^{2}$ when $p \equiv 1,3(\bmod 8)$, with $c$ and $d$ integers. Therefore, for all $n \geq 1, k \geq 3, \beta_{k}(n)$ is an integer.

Corollary 1.6. Let $k \geq 3$ be an odd integer and let $g_{k}(z)=: \sum_{n=1}^{\infty} \beta_{k}(n) q^{n}$ be the weight $k$ CM newform described in Theorem 1.5. Then, for any prime $p>2$ and integer $r \geq 1$,

$\beta_{k}(p)^{r}=\sum_{t=0}^{\left\lfloor\frac{r-1}{2}\right\rfloor}\left(\begin{array}{l}r \\ t\end{array}\right) p^{t(k-1)} \beta_{(r-2 t)(k-1)+1}(p)+ \begin{cases}\left(\begin{array}{l}r \\ \frac{r}{2}\end{array}\right) p^{\frac{r}{2}(k-1)}, & \text { if } p \equiv 1,3(\bmod 8) \text { and } r \text { even }, \\ 0, & \text { otherwise. }\end{cases}$

Also,

$$
\beta_{k}(2)^{r}=\beta_{r(k-1)+1}(2) .
$$

Corollary 1.7. Let $k \geq 3$ be an odd integer and let $g_{k}(z)=: \sum_{n=1}^{\infty} \beta_{k}(n) q^{n}$ be the weight $k C M$ newform described in Theorem 1.5. Then, for any prime $p>2$ and integer $r \geq 1$,

$$
\beta_{k}(p)^{r} \equiv \beta_{r(k-1)+1}(p) \quad\left(\bmod p^{k-1}\right) .
$$

Theorem 1.8. Let $k \geq 3$ be an odd integer and let $g_{k}(z)=: \sum_{n=1}^{\infty} \beta_{k}(n) q^{n}$ be the weight $k$ $C M$ newform described in Theorem 1.5. Let $D(n)$ be the sequence defined in (1.6). Then, for any prime $p>2$ and integer $r \geq 1$,

$$
(-1)^{\frac{p^{r}-1}{2}} D\left(\frac{p^{r}-1}{2}\right) \equiv \beta_{2 r+1}(p) \quad(\bmod p) .
$$

We note that the congruence relation in Theorem 1.8 does not hold modulo $p^{2}$.

Applying the methods used to prove Theorems 1.4 and 1.8, to the results in [13, gives a slight enhancement of (1.2).

Theorem 1.9. Let $k \geq 2$ be an integer and let $h_{k}(z)=: \sum_{n=1}^{\infty} \alpha_{k}(n) q^{n}$ be the weight $k$ $C M$ newform described in Section 1. Let $A(n)$ be the sequence defined in (1.1). Then, for any prime $p \geq 5$ and integer $r \geq 1$,

$$
A\left(\frac{p^{r}-1}{2}\right) \equiv \gamma_{2 r+1}(p) \quad\left(\bmod p^{2}\right) .
$$

\section{Preliminaries}

2.1. Modular forms with complex multiplication and Hecke characters. In this section, we recall some properties of modular forms with complex multiplication and Hecke characters. For more details, see [17].

Suppose $\psi$ is a nontrivial real Dirichlet character with corresponding quadratic field $K$. A newform $f(z)=\sum_{n=1}^{\infty} \gamma(n) q^{n}$, where $q:=e^{2 \pi i z}$, has complex multiplication (CM) by $\psi$, or by $K$, if $\gamma(p)=\psi(p) \gamma(p)$ for all primes $p$ in a set of density one.

By the work of Hecke and Shimura we can construct CM newforms using Hecke characters. Let $K=\mathbb{Q}(\sqrt{-d})$ be an imaginary quadratic field with discriminant $D$, and let $\mathcal{O}_{K}$ be its ring of integers. For an ideal $\mathfrak{f} \in \mathcal{O}_{K}$, let $I(\mathfrak{f})$ denote the group of fractional ideals prime to $\mathfrak{f}$. A Hecke character of weight $k$ and modulo $\mathfrak{f}$ is a homomorphism $\Phi: I(\mathfrak{f}) \rightarrow \mathbb{C}^{*}$, 
satisfying $\Phi\left(\alpha \mathcal{O}_{K}\right)=\alpha^{k-1}$ when $\alpha \equiv^{\times} 1(\bmod \mathfrak{f})$. Let $N(\mathfrak{a})$ denote the norm of the ideal a. Then,

$$
f(z):=\sum_{\mathfrak{a}} \Phi(\mathfrak{a}) q^{N(\mathfrak{a})}=\sum_{n=1}^{\infty} \gamma(n) q^{n},
$$

where the sum is over all ideals $\mathfrak{a}$ in $\mathcal{O}_{K}$ prime to $\mathfrak{f}$, is a Hecke eigenform of weight $k$ on $\Gamma_{0}(|D| \cdot N(\mathfrak{f}))$ with Nebentypus $\chi(n)=\left(\frac{D}{n}\right) \frac{\Phi\left(n \mathcal{O}_{K}\right)}{n^{k-1}}$. Here, $\left(\frac{a}{n}\right)$ is the Kronecker symbol. Furthermore, $f$ has CM by $K$. We call $\mathfrak{f}$ the conductor of $\Phi$ if $\mathfrak{f}$ is minimal, i.e., if $\Phi$ is defined modulo $\mathfrak{f}^{\prime}$ then $\mathfrak{f} \mid \mathfrak{f}^{\prime}$. If $\mathfrak{f}$ is the conductor of $\Phi$ then $f(z)$ is a newform. From [17, we also know that every CM newform comes from a Hecke character in this way.

2.2. The ideals of $\mathbb{Q}(\sqrt{-3})$. Let $K=\mathbb{Q}(\sqrt{-3})$ and let $\omega=\frac{1+\sqrt{-3}}{2}$. The ring of integers of $K$ is $\mathcal{O}_{K}=\mathbb{Z}[\omega]$, which is a principal ideal domain. Therefore, all fractional ideals of $K$ are also principal, and are of the form $\frac{1}{m}(\alpha)$ where $m \in \mathbb{Z} \backslash\{0\}$ and $\alpha \in \mathcal{O}_{K}$.

We will be interested in ideals of $\mathcal{O}_{K}$ of norm $p$, a prime. Let $\mathfrak{I}=(x+y \omega)$ be an ideal of $\mathcal{O}_{K}$. Then $N(\mathfrak{I})=N(x+y \omega)=x^{2}+x y+y^{2}$. If $p=x^{2}+x y+y^{2}=(x-y)^{2}+3 x y$, then $p$ is a square modulo 3 and so $p=3$ or $p \equiv 1(\bmod 6)$. It is well known [10] that a prime $p$ can be written as $p=a^{2}+3 b^{2}, a$ and $b$ integers, uniquely, up to the sign of $a$ and $b$, if and only if $p=3$ or $p \equiv 1(\bmod 6)$. If we let $x=a-b$ and $y=2 b$ then $x^{2}+x y+y^{2}=p$. So there exists an ideal $\mathfrak{I}$ with $N(\mathfrak{I})=p$ if and only if $p=3$ or $p \equiv 1(\bmod 6)$.

Now we determine how many ideals of norm $p$ there are when $p=3$, and when $p \equiv 1$ $(\bmod 6)$. Let $S_{1}=\left\{(a, b) \mid a+3 b^{2}=p\right\}$. We define an equivalence relation, $\sim_{1}$ on $S_{1}$ by

$$
\left(a_{1}, b_{1}\right) \sim_{1}\left(a_{2}, b_{2}\right) \Longleftrightarrow\left|a_{1}\right|=\left|a_{2}\right|,\left|b_{1}\right|=\left|b_{2}\right| .
$$

Then the order of $S_{1} / \sim_{1}$ is one. Next we let $S_{2}$ be the set of all ideals of $\mathcal{O}_{K}$ which have norm $p$. We define an equivalence relation, $\sim_{2}$ on $S_{2}$ by

$$
\left(x_{1}+y_{1} \omega\right) \sim_{2}\left(x_{2}+y_{2} \omega\right) \Longleftrightarrow\left(x_{2}+y_{2} \omega\right) \in\left\{\left(x_{1}+y_{1} \omega\right),\left(y_{1}+x_{1} \omega\right)\right\} .
$$

Note that each equivalence class in $S_{2} / \sim_{2}$ contains at most two elements. We have a bijective map $\phi: S_{1} / \sim_{1} \rightarrow S_{2} / \sim_{2}$ given by $\phi([a, b])=[a-b+2 b \omega]$, with inverse

$$
\phi^{-1}([x+y \omega])= \begin{cases}{\left[x+\frac{y}{2}, \frac{y}{2}\right]} & \text { if } x \text { odd, } y \text { even, } \\ {\left[y+\frac{x}{2}, \frac{x}{2}\right]} & \text { if } x \text { even, } y \text { odd }, \\ {\left[\frac{x-y}{2}, \frac{x+y}{2}\right]} & \text { if } x \text { odd, } y \text { odd } .\end{cases}
$$

Therefore $S_{2} / \sim_{2}$ contains only one equivalence class. The group of units of $\mathcal{O}_{K}$ is cyclic of order 6 generated by $\omega$. It is easy then to check that $(x+y \omega)=(y+x \omega)$ only if $p=3$, when $x=y$. Hence, when $p=3$ there is only one ideal of norm $p$ and when $p \equiv 1(\bmod 6)$ there are two ideals of norm $p$. Later, it will be convenient for us to choose the representatives of these ideal as $-1+2 \omega=\sqrt{-3}$ in the case that $p=3$, and, $(a-b)+2 b \omega=a+b \sqrt{-3}$ and $(a+b)-2 b \omega=a-b \sqrt{-3}$ in the case $p \equiv 1(\bmod 6)$, where $p=a^{2}+3 b^{2}$ with $a \equiv 1$ $(\bmod 3)$ and $b>0$. 
2.3. The Apéry-like numbers. The $A(n)$ Apéry numbers defined in (1.1) satisfy the three term recurrence relation

$$
b(n+1)^{2} u(n+1)+\left(a n^{2}+a n-\lambda\right) u(n)+n^{2} u(n-1)=0,
$$

when $(a, b, \lambda)=(11,-1,-3)$ and $u(0)=1$. Following Beukers [6], Zagier [20] conducted a search involving over 100 million suitable triples $(a, b, \lambda)$ and found 36 triples which yielded an integral solution to (2.1) with $u(0)=1$. Six of these solutions are classed as sporadic and all six have binomial sum representations. The sequences $C(n)$ and $D(n)$, defined in (1.5) and (1.6), are two of these sporadic cases. The $B(n)$ Apéry numbers also satisfy a three term recurrence relation and generalized searches similar to Zagier's have been conducted for this relation also [3, 9]. In general, integer sequences which are solutions to either of these recurrences are known as Apéry-like numbers. Congruence properties of many of these sequences have been studied by various authors $[8,11,12,14,15,16,18,19$.

Of particular interest to us are the results in [18. For $M \in\{2,3,4\}$, let $U_{M}(n)$ be the sequences defined by $U_{2}(n)=(-1)^{\frac{n-1}{2}} D\left(\frac{n-1}{2}\right), U_{3}(n)=C\left(\frac{n-1}{2}\right)$, and $U_{4}(n)=$ $(-1)^{\frac{n-1}{2}} A\left(\frac{n-1}{2}\right)$ when $n$ is odd, and $U_{M}(n)=0$ when $n$ is even. Then for primes $p$ not dividing $M$ and all positive integers $m$ and $r$, we have

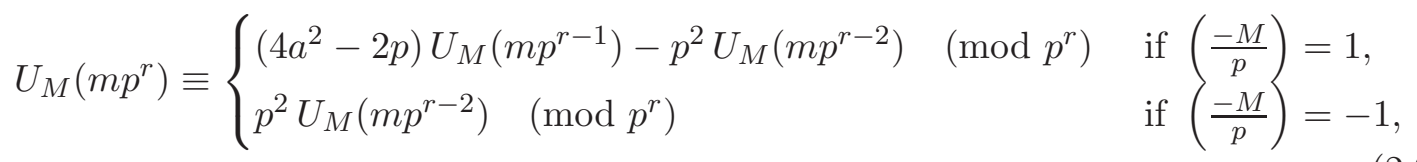

where $p=a^{2}+M b^{2}, a$ and $b$ integers, when $\left(\frac{-M}{p}\right)=1$. We will see later that $4 a^{2}-2 p$ equals $\beta_{3}(p), \gamma_{3}(p)$ and $\alpha_{3}(p)$ when $M=2,3$ and 4 respectively. Thus, (2.2) gives us a congruence relation between values of $D(n), C(n)$, and $A(n)$ and the Fourier coefficients of $g_{3}(z), f_{3}(z)$, and $h_{3}(z)$ respectively, which will be central to our proofs of Theorems 1.4, 1.8 and 1.9 .

\section{Proofs}

Proof of Theorem 1.1. For $k$ in each equivalence class modulo 6, we will define a Hecke character $\Psi_{k}$ and construct the required CM newform $f_{k}$, using the methodology outlined in Section 2.1.

For an ideal $\mathfrak{f} \in \mathcal{O}_{K}$, let $I(\mathfrak{f})$ denote the group of fractional ideals prime to $\mathfrak{f}$, and let $J(\mathfrak{f})$ be the subset of principal fractional ideals whose generator is multiplicatively congruent to 1 modulo $\mathfrak{f}$, i.e., $J(\mathfrak{f})=\left\{(\alpha) \in I(\mathfrak{f}) \mid \alpha \equiv^{\times} 1(\bmod \mathfrak{f})\right\}$.

Let $K=\mathbb{Q}(\sqrt{-3})$, which has discriminant $D=-3$ and whose ring of integers is $\mathcal{O}_{K}=\mathbb{Z}\left[\frac{1+\sqrt{-3}}{2}\right]$, which is a principal ideal domain. Therefore, all fractional ideals of $K$ are also principal, and are of the form $\frac{1}{m}(\alpha)$ where $m \in \mathbb{Z} \backslash\{0\}$ and $\alpha \in \mathcal{O}_{K}$.

We let $\omega:=\frac{1+\sqrt{-3}}{2}$ for brevity. In what follows, $p$ will always denote a prime. When $p \equiv 1(\bmod 6)$ we will reserve $a$ and $b$ exclusively to be the integers defined by $p=a^{2}+3 b^{2}$ with $a \equiv 1(\bmod 3)$ and $b>0$. Note that $a$ and $b$ must have different parity. We also note the discussion in Section 2.2. where we express the ideals of $\mathcal{O}_{K}$ which have norm $p \equiv 1$ $(\bmod 6)$ in terms of $a, b$ and $\omega$. 
Case $1: k \equiv 1(\bmod 6)$. Let $\mathfrak{f}=(1)$. Then $I(\mathfrak{f})=J(\mathfrak{f})$ is the set of all fractional ideals. We define the Hecke character $\Phi_{k}: I((1)) \rightarrow \mathbb{C}^{*}$ of weight $k$ and conductor (1) by

$$
\Phi_{k}\left(\frac{1}{m}(\alpha)\right)=\left(\frac{\alpha}{m}\right)^{k-1} .
$$

Therefore,

$$
f_{k}(z):=\sum_{\mathfrak{a}} \Phi_{k}(\mathfrak{a}) q^{N(\mathfrak{a})}=\sum_{n=1}^{\infty} \gamma_{k}(n) q^{n} \in S_{k}\left(\Gamma_{0}(3),\left(\frac{-3}{\cdot}\right)\right)
$$

is a CM newform and, for $p$ an odd prime,

$$
\gamma_{k}(p)= \begin{cases}(a-b+2 b \omega)^{k-1}+(a+b-2 b \omega)^{k-1} & \text { if } p \equiv 1(\bmod 6) \\ (-3)^{\frac{k-1}{2}} & \text { if } p=3 \\ 0 & \text { otherwise. }\end{cases}
$$

Note here that $(-1+2 \omega)^{2}=-3$.

Case $2: k \equiv 4(\bmod 6)$. Let $\mathfrak{f}=(1+\omega)$. Then

$$
I((1+\omega))=\left\{\frac{1}{m}(\alpha) \mid \alpha=x+y \omega, x+2 y \not \equiv 0(\bmod 3), m \not \equiv 0(\bmod 3)\right\}
$$

and

$$
J((1+\omega))=\left\{\frac{1}{m}(\alpha) \mid \alpha=x+y \omega, x+2 y \equiv m \not \equiv 0(\bmod 3)\right\} .
$$

We define the Hecke character $\Phi_{k}: I((1+\omega)) \rightarrow \mathbb{C}^{*}$ of weight $k$ and conductor $(1+\omega)$ by

$$
\Phi_{k}\left(\frac{1}{m}(x+y \omega)\right)=\left(\frac{x+y \omega}{m}\right)^{k-1} \chi\left(\frac{1}{m}(x+y \omega)\right),
$$

where

$$
\chi\left(\frac{1}{m}(x+y \omega)\right)= \begin{cases}+1, & \text { if } x+2 y \equiv m(\bmod 3), \\ -1, & \text { if } x+2 y \neq \equiv m(\bmod 3) .\end{cases}
$$

Therefore,

$$
f_{k}(z):=\sum_{\mathfrak{a}} \Phi_{k}(\mathfrak{a}) q^{N(\mathfrak{a})}=\sum_{n=1}^{\infty} \gamma_{k}(n) q^{n} \in S_{k}\left(\Gamma_{0}(9)\right)
$$

is a CM newform, and for $p$ an odd prime,

$$
\gamma_{k}(p)= \begin{cases}(a-b+2 b \omega)^{k-1}+(a+b-2 b \omega)^{k-1} & \text { if } p \equiv 1(\bmod 6) \\ 0 & \text { otherwise }\end{cases}
$$

Case $3: k \equiv 3,5(\bmod 6)$. Let $\mathfrak{f}=(2)$. Then

$$
I((2))=\left\{\frac{1}{m}(\alpha) \mid \alpha=x+y \omega, x \text { and } y \text { not both even, } m \text { odd }\right\}
$$

and

$$
J((2))=\left\{\frac{1}{m}(\alpha) \mid \alpha=x+y \omega, x \text { odd and } y \text { even, } m \text { odd }\right\} .
$$

We define the Hecke character $\Phi_{k}: I((2)) \rightarrow \mathbb{C}^{*}$ of weight $k$ and conductor (2) by

$$
\Phi_{k}\left(\frac{1}{m}(x+y \omega)\right)=\left(\frac{x+y \omega}{m}\right)^{k-1} \psi((x+y \omega))^{k-1},
$$

where

$$
\psi((x+y \omega))= \begin{cases}1 & \text { if } x \text { odd, } y \text { even } \\ \omega^{2} & \text { if } x \text { even, } y \text { odd } \\ \omega^{4} & \text { if } x \text { odd, } y \text { odd }\end{cases}
$$


Therefore,

$$
f_{k}(z):=\sum_{\mathfrak{a}} \Phi_{k}(\mathfrak{a}) q^{N(\mathfrak{a})}=\sum_{n=1}^{\infty} \gamma_{k}(n) q^{n} \in S_{k}\left(\Gamma_{0}(12),\left(\frac{-3}{\cdot}\right)\right)
$$

is a CM newform and, for $p$ an odd prime,

$$
\gamma_{k}(p)= \begin{cases}(a-b+2 b \omega)^{k-1}+(a+b-2 b \omega)^{k-1} & \text { if } p \equiv 1(\bmod 6), \\ (-3)^{\frac{k-1}{2}} & \text { if } p=3 \\ 0 & \text { otherwise. }\end{cases}
$$

Case $4: k \equiv 0,2(\bmod 6)$. Let $\mathfrak{f}=(2+2 \omega)$. Then

$I((2+2 \omega))=\left\{\frac{1}{m}(\alpha) \mid \alpha=x+y \omega, x\right.$ and $y$ not both even, $x+2 y \not \equiv 0(\bmod 3), m$ odd,$\left.m \not \equiv 0(\bmod 3)\right\}$ and

$$
J((2+2 \omega))=\left\{\frac{1}{m}(\alpha) \mid \alpha=x+y \omega, x \text { odd and } y \text { even, } m \text { odd }, x+2 y \equiv m \not \equiv 0(\bmod 3)\right\} .
$$

We define the Hecke character $\Phi_{k}: I((2+2 \omega)) \rightarrow \mathbb{C}^{*}$ of weight $k$ and conductor $(2+2 \omega)$ by

$$
\Phi_{k}\left(\frac{1}{m}(x+y \omega)\right)=\left(\frac{x+y \omega}{m}\right)^{k-1} \chi\left(\frac{1}{m}(x+y \omega)\right) \psi((x+y \omega))^{k-1},
$$

where $\chi(\cdot)$ and $\psi(\cdot)$ are defined as in (3.1) and (3.2). Therefore,

$$
f_{k}(z):=\sum_{\mathfrak{a}} \Phi_{k}(\mathfrak{a}) q^{N(\mathfrak{a})}=\sum_{n=1}^{\infty} \gamma_{k}(n) q^{n} \in S_{k}\left(\Gamma_{0}(36)\right)
$$

is a CM newform, and for $p$ an odd prime,

$$
\gamma_{k}(p)= \begin{cases}(a-b+2 b \omega)^{k-1}+(a+b-2 b \omega)^{k-1} & \text { if } p \equiv 1(\bmod 6) \\ 0 & \text { otherwise. }\end{cases}
$$

So now we have proved all but the last line of the theorem. By the multiplicative properties of the Fourier coefficients of newforms it suffices to show that $\gamma_{k}(p)$ is an integer for all primes $p$. This is obvious when $p \not \equiv 1(\bmod 6)$. We now examine the case when $p \equiv 1$ $\bmod 6$. We have shown that for all $k$,

$$
\begin{aligned}
\gamma_{k}(p)= & (a+b \sqrt{-3})^{k-1}+(a-b \sqrt{-3})^{k-1} \\
= & \sum_{t=0}^{k-1}\left(\begin{array}{c}
k-1 \\
t
\end{array}\right) a^{k-1-t} b^{t}(\sqrt{-3})^{t}+\sum_{t=0}^{k-1}\left(\begin{array}{c}
k-1 \\
t
\end{array}\right) a^{k-1-t}(-1)^{t} b^{t}(\sqrt{-3})^{t} \\
= & \sum_{t=0}^{k-1}\left(\begin{array}{c}
k-1 \\
t
\end{array}\right) a^{k-1-t} b^{t}(\sqrt{-3})^{t}\left[1+(-1)^{t}\right] \\
= & \sum_{\substack{t=0 \\
\text { teven }}}^{k-1}\left(\begin{array}{c}
k-1 \\
t
\end{array}\right) a^{k-1-t} b^{t}(-3)^{\frac{t}{2}},
\end{aligned}
$$

which is an integer 
Proof of Corollary 1.2. For $p=3$ the result follows directly from the formula for $\gamma_{k}(3)$ in Theorem 1.1. When $p \equiv 5(\bmod 6)$, both sides equal zero, as $\gamma_{k}(p)=0$ for all $k \geq 2$. We now assume $p \equiv 1(\bmod 6)$. Let $\gamma^{+}(p)=a+b \sqrt{-3}$ and $\gamma^{-}(p)=a-b \sqrt{-3}$. From Theorem 1.1 we get that

$$
\begin{aligned}
& \gamma_{k}(p)^{r}=\left(\gamma^{+}(p)^{k-1}+\gamma^{-}(p)^{k-1}\right)^{r} \\
& =\sum_{t=0}^{r}\left(\begin{array}{l}
r \\
t
\end{array}\right) \gamma^{+}(p)^{t(k-1)} \gamma^{-}(p)^{(r-t)(k-1)} \\
& =\sum_{t=0}^{\left\lfloor\frac{r-1}{2}\right\rfloor}\left(\begin{array}{l}
r \\
t
\end{array}\right)\left(\gamma^{+}(p)^{r(k-1)} \gamma^{-}(p)^{(r-t)(k-1)}+\gamma^{+}(p)^{(r-t)(k-1)} \gamma^{-}(p)^{r(k-1)}\right) \\
& + \begin{cases}\left(\begin{array}{l}
r \\
\frac{r}{2}
\end{array}\right)\left(\gamma^{+}(p) \gamma^{-}(p)\right)^{\frac{r}{2}(k-1)}, & \text { if } r \text { is even, } \\
0, & \text { if } r \text { is odd. }\end{cases}
\end{aligned}
$$

To complete the proof, we write the summand as

$$
\left(\begin{array}{l}
r \\
t
\end{array}\right)\left(\gamma^{+}(p) \gamma^{-}(p)\right)^{t(k-1)}\left(\gamma^{-}(p)^{(r-2 t)(k-1)}+\gamma^{+}(p)^{(r-2 t)(k-1)}\right)
$$

and note that

$$
\gamma^{-}(p)^{(r-2 t)(k-1)}+\gamma^{+}(p)^{(r-2 t)(k-1)}=\gamma_{(r-2 t)(k-1)+1}(p)
$$

and that $\gamma^{+}(p) \gamma^{-}(p)=p$.

Proof of Corollary 1.3. This follows from reducing the result in Corollary 1.2 modulo $p^{k-1}$.

Proof of Theorem 1.4. Let $p$ be prime. When $p \equiv 1(\bmod 6)$, let $p=a^{2}+3 b^{2}$, with $a \equiv 1(\bmod 3)$ and $b>0$ integers. We first show that $4 a^{2}-2 p=\gamma_{3}(p)$, the $p$-th Fourier coefficient of the weight three CM form $f_{3}(z)$ constructed in Theorem 1.1. From Theorem 1.1 we get that

$$
\begin{aligned}
\gamma_{3}(p) & =(a+b \sqrt{-3})^{2}+(a-b \sqrt{-3})^{2} \\
& =2 a^{2}-6 b^{2} \\
& =4 a^{2}-2 p .
\end{aligned}
$$

Then, taking $m=r=1$ in (2.2), and using the facts that $U_{3}(1)=1$ and $\gamma_{3}(p)=0$ when $p \equiv 5(\bmod 6)$, we see that for all primes $p>3$,

$$
U_{3}(p) \equiv \gamma_{3}(p) \quad(\bmod p) .
$$

Substituting (3.3) back into (2.2) with $m=1$ and reducing modulo $p$ we have

$$
U_{3}\left(p^{r}\right) \equiv U_{3}(p) U_{3}\left(p^{r-1}\right) \quad(\bmod p)
$$

for all positive integers $r$. Using a simple inductive argument we then get that for all primes $p>3$ and integers $r \geq 1$,

$$
U_{3}\left(p^{r}\right) \equiv U_{3}(p)^{r} \quad(\bmod p) .
$$


Accounting for (3.3) yields

$$
U_{3}\left(p^{r}\right) \equiv \gamma_{3}(p)^{r} \quad(\bmod p),
$$

which completes the proof as $U\left(p^{r}\right)=C\left(\frac{p^{r}-1}{2}\right)$ and $\gamma_{3}(p)^{r} \equiv \gamma_{2 r+1}(p)(\bmod p)$ by Corollary 1.3. It is worth noting also that (3.4) gives us the nice relation

$$
C\left(\frac{p^{r}-1}{2}\right) \equiv C\left(\frac{p-1}{2}\right)^{r}(\bmod p) .
$$

Proof of Theorem 1.5. Let $K=\mathbb{Q}(\sqrt{-2})$, which has discriminant $D=-8$ and whose ring of integers is $\mathcal{O}_{K}=\mathbb{Z}[\sqrt{-2}]$, which is a principal ideal domain. Therefore, all fractional ideals of $K$ are also principal, and are of the form $\frac{1}{m}(\alpha)$ where $m \in \mathbb{Z} \backslash\{0\}$ and $\alpha \in \mathcal{O}_{K}$.

It is well known [10] that a prime $p$ can be written as $p=c^{2}+2 d^{2}, c$ and $d$ integers, uniquely, up to the sign of $c$ and $d$, if and only if $p=2$ or $p \equiv 1$ or $3(\bmod 8)$. Let $\mathfrak{I}=(c+d \sqrt{-2})$ be an ideal of $\mathcal{O}_{K}$. Then $N(\mathfrak{I})=c^{2}+2 d^{2}$. The units of $\mathcal{O}_{K}$ are \pm 1 , and therefore, for a prime $p$ there are two ideals of norm $p$ when $p \equiv 1,3(\bmod 8)$ and one when $p=2$.

Let $\mathfrak{f}=(1)$. Then $I(\mathfrak{f})=J(\mathfrak{f})$ is the set of all fractional ideals. We define the Hecke character $\Phi_{k}: I((1)) \rightarrow \mathbb{C}^{*}$ of weight $k$ and conductor (1) by

$$
\Phi_{k}\left(\frac{1}{m}(\alpha)\right)=\left(\frac{\alpha}{m}\right)^{k-1} .
$$

Therefore,

$$
g_{k}(z):=\sum_{\mathfrak{a}} \Phi_{k}(\mathfrak{a}) q^{N(\mathfrak{a})}=\sum_{n=1}^{\infty} \beta_{k}(n) q^{n} \in S_{k}\left(\Gamma_{0}(8),\left(\frac{-8}{\cdot}\right)\right)
$$

is a CM newform and, for $p$ a prime,

$$
\beta_{k}(p)= \begin{cases}(c+d \sqrt{-2})^{k-1}+(c-d \sqrt{-2})^{k-1} & \text { if } p \equiv 1,3(\bmod 8) \\ (-2)^{\frac{k-1}{2}} & \text { if } p=2 \\ 0 & \text { otherwise. }\end{cases}
$$

We now note that when $p \equiv 1,3(\bmod 8)$,

$$
\begin{aligned}
\beta_{k}(p) & =(c+d \sqrt{-2})^{k-1}+(c-d \sqrt{-2})^{k-1} \\
& \left.=\sum_{t=0}^{k-1}\left(\begin{array}{c}
k-1 \\
t
\end{array}\right) c^{k-1-t} d^{t}(\sqrt{-2})^{t}\left[1+(-1)^{t}\right)\right] \\
& =\sum_{\substack{t=0 \\
\text { teven }}}^{k-1}\left(\begin{array}{c}
k-1 \\
t
\end{array}\right) c^{k-1-t} d^{t}(-2)^{\frac{t}{2}}
\end{aligned}
$$

which is an integer.

Proof of Corollary 1.6. Proceed along the same lines as in the proof of Corollary 1.2 but with $\beta^{+}(p)=(c+d \sqrt{-2})$ and $\beta^{-}(p)=(c-d \sqrt{-2})$. 
Proof of Corollary 1.7. This follows from reducing the result in Corollary [1.6 modulo $p^{k-1}$.

Proof of Theorem 1.8. Let $p$ be prime. When $p \equiv 1,3(\bmod 8)$, let $p=c^{2}+3 d^{2}$, with $c$ and $d$ integers. From Theorem 1.5 we get that

$$
\left.\beta_{3}(p)=(c+d \sqrt{-2})\right)^{2}+(c-d \sqrt{-2})^{2}=2 a^{2}-4 b^{2}=4 a^{2}-2 p .
$$

Then, taking $m=r=1$ in (2.2), and using the facts that $U_{2}(1)=1$ and $\beta_{3}(p)=0$ when $p \equiv 5,7(\bmod 8)$, we see that for all primes $p>2$,

$$
U_{2}(p) \equiv \beta_{3}(p) \quad(\bmod p) .
$$

Substituting (3.5) back into (2.2) with $m=1$ and reducing modulo $p$ we have

$$
U_{2}\left(p^{r}\right) \equiv U_{2}(p) U_{2}\left(p^{r-1}\right) \quad(\bmod p)
$$

for all positive integers $r$. Using a simple inductive argument we then get that for all primes $p>2$ and integers $r \geq 1$,

$$
U_{2}\left(p^{r}\right) \equiv U_{2}(p)^{r} \quad(\bmod p) .
$$

Accounting for (3.5) yields

$$
U_{2}\left(p^{r}\right) \equiv \beta_{3}(p)^{r} \quad(\bmod p),
$$

which completes the proof as $U_{2}\left(p^{r}\right)=(-1)^{\frac{p^{r}-1}{2}} D\left(\frac{p^{r}-1}{2}\right)$ and $\beta_{3}(p)^{r} \equiv \beta_{2 r+1}(p)(\bmod p)$ by Corollary 1.7. It is worth noting also that (3.6) gives us the nice relation

$$
D\left(\frac{p^{r}-1}{2}\right) \equiv D\left(\frac{p-1}{2}\right)^{r} \quad(\bmod p)
$$

as $(-1)^{\frac{p^{r}-1}{2}}=(-1)^{\frac{r(p-1)}{2}}$.

Proof of Theorem 1.9. Following the description of $h_{k}(z)$ in Section 1, we note that $\alpha_{3}(p)=$ $4 a^{2}-2 p$ when $p \equiv 1(\bmod 4)$. Note also that, for $p \geq 5$, Ahlgren's result (1.2) gives us

$$
(-1)^{\frac{(p-1)}{2}} U_{4}(p)=A\left(\frac{p-1}{2}\right) \equiv \alpha_{3}(p) \quad\left(\bmod p^{2}\right) .
$$

So, considering (2.2) modulo $p^{2}$ with $m=1$ and $r \geq 2$ we get that

$$
U_{4}\left(p^{r}\right) \equiv U_{4}(p) U_{4}\left(p^{r-1}\right) \quad\left(\bmod p^{2}\right),
$$

which holds for all $p \geq 5$, as $\alpha_{3}(p)=0$ when $p \equiv 3(\bmod 4)$, and $(-1)^{\frac{(p-1)}{2}}=1$ when $p \equiv 1(\bmod 4)$. Using a simple inductive argument we then get that for all primes $p \geq 5$ and integers $r \geq 2$,

$$
U_{2}\left(p^{r}\right) \equiv U_{2}(p)^{r} \quad\left(\bmod p^{2}\right)
$$

which implies

$$
(-1)^{\frac{\left(p^{r}-1\right)}{2}} A\left(\frac{p^{r}-1}{2}\right) \equiv \alpha_{3}(p)^{r} \quad\left(\bmod p^{2}\right) .
$$

This completes the proof as $\alpha_{3}(p)^{r} \equiv \alpha_{2 r+1}(p)\left(\bmod p^{2}\right)$ from $(1.3)$, and $(-1)^{\frac{\left(p^{r}-1\right)}{2}}=1$ when $p \equiv 1(\bmod 4)$. 


\section{ACKNOWLEDGEMENTS}

The second author is supported by a grant from the Simons Foundation (\#353329, Dermot McCarthy).

\section{REFERENCES}

[1] S. Ahlgren, Gaussian hypergeometric series and combinatorial congruences, Symbolic computation, number theory, special functions, physics and combinatorics (Gainesville, FL, 1999), 1-12, Dev. Math., 4, Kluwer Acad. Publ., Dordrecht, 2001.

[2] S. Ahlgren, K. Ono, A Gaussian hypergeometric series evaluation and Apéry number congruences, J. reine angew. Math. 518 (2000), 187-212.

[3] G. Almkvist, W. Zudilin, Differential equations, mirror maps and zeta values Mirror symmetry. V, 481-515, AMS/IP Stud. Adv. Math., 38, Amer. Math. Soc., Providence, RI, 2006.

[4] R. Apéry, Irrationalit de $\zeta(2)$ et $\zeta(3)$ Astérisque No. 61 (1979), 11-13.

[5] F. Beukers, A note on the irrationality of $\zeta(2)$ and $\zeta(3)$, Bull. London Math. Soc. 11 (1979), no. 3, 268-272.

[6] F. Beukers, On Dwork's accessory parameter problem, Math. Z. 241 (2002), no. 2, 425 ?444.

[7] F. Brown, Irrationality proofs for zeta values, moduli spaces and dinner parties, Mosc. J. Comb. Number Theory 6 (2016), no. 2-3, 102-165.

[8] H. Chan, S. Cooper, F. Sica, Congruences satisfied by Apéry-like numbers, Int. J. Number Theory 6 (2010), no. 1, 89-97.

[9] S. Cooper Sporadic sequences, modular forms and new series for 1/ $\pi$, Ramanujan J. 29 (2012), no. $1-3,163 ? 183$.

[10] D. Cox, Primes of the form x2+ny2, Fermat, class field theory and complex multiplication. A WileyInterscience Publication. John Wiley \& Sons, Inc., New York, 1989.

[11] E. Delaygue, Arithmetic properties of Apéry-like numbers, Compos. Math. 154 (2018), no. 2, 249274.

[12] A. Malik, A. Straub, Divisibility properties of sporadic Apéry-like numbers, Res. Number Theory 2 (2016), Art. 5, 26 pp.

[13] D. McCarthy, R. Osburn, A. Straub, Sequences, modular forms and cellular integrals, arXiv:1705.05586.

[14] R. Osburn, B. Sahu, Supercongruences for Apéry-like numbers Adv. in Appl. Math. 47 (2011), no. 3, 631-638.

[15] R. Osburn, B. Sahu Congruences via modular forms, Proc. Amer. Math. Soc. 139 (2011), no. 7, $2375-2381$.

[16] R. Osburn, B. Sahu, A. Straub, Supercongruences for sporadic sequences, Proc. Edinb. Math. Soc. (2) 59 (2016), no. 2, 503-518.

[17] K. Ribet, Galois representations attached to eigenforms with Nebentypus, Modular functions of one variable, V (Proc. Second Internat. Conf., Univ. Bonn, Bonn, 1976), pp. 17-51. Lecture Notes in Math., Vol. 601, Springer, Berlin, 1977.

[18] J. Stienstra, F. Beukers, On the Picard-Fuchs equation and the formal Brauer group of certain elliptic K3-surfaces, Math Ann. 271 (1985) 269-304.

[19] H. Verrill Congruences related to modular forms, Int. J. Number Theory 6 (2010), no. 6, 1367-1390.

[20] D. Zagier, Integral solutions of Apéry-like recurrence equations, Groups and symmetries, 349-366, CRM Proc. Lecture Notes, 47, Amer. Math. Soc., Providence, RI, 2009. USA

Department of Mathematics \& Statistics, Texas Tech University, Lubbock, TX 79410-1042,

E-mail address: Alexis.Gomez@ttu.edu

E-mail address: dermot.mccarthy@ttu.edu

E-mail address: Dylan. Young@ttu.edu 